\title{
FINAL SETS FOR OPERATORS ON REAL ENTIRE FUNCTIONS OF ORDER ONE, NORMAL TYPE
}

\author{
C. L. PRATHER ${ }^{1}$
}

\begin{abstract}
Let $f$ be a real entire function of order one, normal type that is bounded on the real axis and $L=\varphi(D), D=(d / d z)$ with $\varphi(\omega)$ a Laguerre-Pólya function satisfying $\varphi(0)=0$. Then the final set of $f$ with respect to $L$ is contained in the real axis as either a discrete subset or the whole axis.
\end{abstract}

1. Introduction. We start with the statement of our result.

THEOREM. Let $f(z)$ be a real entire function (that is, real valued for $z$ real) of order one, normal type $\lambda$, satisfying $|f(x)| \leqslant 1$ on $(-\infty, \infty)$. Let $L=\varphi(D)$, where $D=$ $(d / d z)$ and $\varphi(\omega)$ is a Laguerre-Pólya function with $\varphi(0)=0$. Then the final set of $f$ with respect to the operator $L$ is either a discrete set on the real axis or the whole real axis depending on whether the number $\beta, \beta=\arg (\varphi(i \lambda) / \varphi(-i \lambda))$ is or is not a rational multiple of $\pi$.

We now define the terms used in the statement of the Theorem. A Laguerre-Pólya function $\varphi$ is one having the well-known form

$$
\varphi(z)=z^{p} \exp \left(-a z^{2}+b z\right) \prod_{n}\left(1-\frac{z}{\alpha_{n}}\right) \exp \left(\frac{z}{\alpha_{n}}\right),
$$

where $a \geqslant 0, b$ and $\alpha_{n}$ are real, $p$ is a nonnegative integer and $\sum_{n}\left(\alpha_{n}\right)^{-2}<\infty$. Next, let $f$ be analytic on a domain $D$ and let $L$ be a differential operator. The final set $S(f, L)$ of the function $f$ with respect to the operator $L$ is defined as follows: A point $z \in S(f, L)$ if and only if every neighborhood of $z$ contains zeros of infinitely many iterates $\left(L^{n} f\right)(z), z \in D$, with $L^{0}=I$ and $L^{n}=L\left(L^{n-1}\right)$, for $n \geqslant 1$.

For derivatives, this notion is classical and is discussed by Pólya in his survey paper [8]. The latest contribution to final sets for derivatives appears to be the very nice results of Gethner [6].

The reader should note that no assumptions are made on the zeros of $f(z)$. When specialized to differentiation with $\varphi(z)=z$, our result shows that the final set is a discrete set on the real axis. Our result illustrates the contrasts that take place between the behavior of zeros of derivatives and the behavior of zeros of iterates of other differential operators. One might compare this result to Pólya's hypothetical

Received by the editors May 5, 1983.

1980 Mathematics Subject Classification. Primary 30A66; Secondary 30A64.

Key words and phrases. Real entire function, exponential type, final set, differential operator.

'The author thanks the National Research Council for its financial support. 
theorems $\mathrm{A}$ and $\mathrm{C}$ in his famous survey paper [8, p. 182]. While these hypothetical theorems remain unsolved, we state them for reference.

A. If the order of the real entire function $f(z)$ is less than 2 and $f(z)$ has only a finite number of complex zeros, then its derivatives, from a certain one onwards, will have no complex zeros at all.

C. If a real entire function $f$ of order greater than one remains bounded for real values of the variable, then its final set contains the whole real axis.

In statement $\mathrm{A}$, by complex zeros Pólya is referring to nonreal zeros. In both statements $\mathrm{A}$ and $\mathrm{C}$, Pólya is referring to behavior with respect to the derivative operator. Contributions to A were made by various people, the strongest result due to Pólya [9], who proved this result for real entire functions of order less than 4/3. The best results in the direction of statement $\mathrm{C}$ were given by $\mathrm{A}$. Edrei [5].

The present paper is a first attempt to understand how Pólya's hypothetical theorems are altered when differentiation is replaced by more general differential operators. A recent survey on zeros of operators on functions and their analytic character may be found in [13].

Our theorem applies to Bessel functions having the form $f(z)=(z / 2)^{-v} J_{v^{v}}(z)$, for $v \geqslant-1 / 2$, which are real entire functions of order one, normal type. They are also bounded on the real axis. The result also applies to real entire functions $f(z)$ which are of order one, normal type $\lambda$, satisfying $f(x) \in L^{p}(-\infty, \infty), 1<p<\infty$. For, by the Paley-Wiener theorem,

$$
f(z)=\int_{-\lambda}^{\lambda} e^{i z t} \psi(t) d t
$$

where $\psi(t) \in L^{q}(-\lambda, \lambda), p^{-1}+q^{-1}=1[1$, Theorem 6.8.13]. That these functions are bounded on the real axis holds by Hölder's inequality.

The result given here is suggested by previous results $[3,10,11]$, where final sets were determined for trigonometric polynomials and finite Fourier transforms that are entire of order one, normal type. For some entire functions having order $\rho \in(1,2)$ that are expressible as a Fourier integral, final sets for some differential operators were determined in [12].

2. Preliminary lemmas. To establish the result we make use of various facts. The first is a very interesting result of Duffin and Schaeffer [4] which allows us to express a real entire function of order one, normal type which is bounded on the real axis as the difference of two other functions that we can handle.

LEMMA 1. Let $f(z)$ be entire of order one, normal type $\lambda$, real valued on the real axis, satisfying $|f(x)| \leqslant 1$ for all real $x$. Then for any real $\alpha$ the function

$$
2 g(z)=\cos (\lambda z+\alpha)-f(z)
$$

is either identically zero or has only real zeros, in fact infinitely many.

In fact, Duffin and Schaeffer show that the real zeros are simple at the points where the strict inequality $|f(x)|<1$ is satisfied. 
The next result is a rather extensive generalization to operators of Bernstein's inequality [1, Theorem 11.1.2]. In its most general form, this generalization is due to B. Ja. Levin [7, Chapter 9]. Instead of presenting it in its most general form, we state it in a form convenient for our use.

Lemma 2 [7, Chapter 9, $\S 3,4]$. Let $L=\varphi(D), D=(d / d z)$ with $\varphi(\omega)$ a Laguerre-Pólya function, i.e. has the form (1.1). Let $g(z)$ be an entire function of exponential type $\sigma$ which is real valued on the real axis and has only real zeros. Let $\omega(z)$ be an entire function of exponential type $\tau \geqslant \sigma$ having no zeros in $\operatorname{Im}(z)<0$ and satisfing

$$
|\omega(z)| \leqslant|\bar{\omega}(z)|
$$

for $\operatorname{Im}(z)>0$. Then

$$
|g(z)| \leqslant|\omega(z)|
$$

for $\operatorname{Im}(z) \leqslant 0$ implies

$$
\left|\left(L^{m} g\right)(z)\right| \leqslant\left|\left(L^{m} \omega\right)(z)\right|
$$

for $\operatorname{Im}(z) \leqslant 0$ and $m=1,2,3, \ldots$

Here $\bar{\omega}$ denotes the function whose power series coefficients are the conjugates of those of $\omega$.

We next treat the case of equality at a point in Lemma 2.

LEMMa 3. Under the assumptions of Lemma 2, assume $\varphi(0)=0$ and $\omega(z)$ is not a polynomial. If there exists a point $z_{0}$ for which $\operatorname{Im}\left(z_{0}\right) \leqslant 0$ and some nonnegative integer $M$ for which

$$
\left|\left(L^{M} g\right)\left(z_{0}\right)\right|=\left|\left(L^{M} \omega\right)\left(z_{0}\right)\right|,
$$

then for all integers $m \geqslant M$,

$$
\left(L^{m} g\right)(z)=C_{1}\left(L^{m} \omega\right)(z)+C_{2}\left(L^{m} \bar{\omega}\right)(z)
$$

for complex numbers $C_{1}, C_{2}$ satisfying $\left|C_{1}\right|+\left|C_{2}\right|=1$.

Proof. Let $\varphi$ have a zero of order $p$ at the origin. Write $L=D^{p} L_{1}$, with $D=(d / d z)$. Condition (2.5) is equivalent to

$$
\left|D^{p M}\left(L_{1}^{M} g\right)\left(z_{0}\right)\right|=\left|D^{p M}\left(L_{1}^{M} \omega\right)\left(z_{0}\right)\right| .
$$

Now, $\left(L_{1}^{M} g\right)(z)$ and $\left(L_{1}^{M} \omega\right)(z)$ are functions that belong to the class $P^{*}$ (see [7, Chapter $8, \S 1$ ] for the definition). That this is true follows from the fact that the functions $g$ and $\omega$ belong to the class $P \subset P^{*}[7$, Chapter 7, §4] and that the operator $L_{1}^{M}$ preserves the class $P^{*}$. Therefore, we apply a known theorem of Levin [7, Chapter 9, Theorem 6] to conclude that

$$
\left(L_{1}^{M} g\right)(z)=C_{1}\left(L_{1}^{M} \omega\right)(z)+C_{2}\left(L_{1}^{M} \bar{\omega}\right)(z)
$$

for complex constants $C_{1}, C_{2}$ satisfying $\left|C_{1}\right|+\left|C_{2}\right|=1$. Then for any integer $m \geqslant M$, we apply the derivative operator to $(2.8)$ and get

$$
\left(L^{m} g\right)(z)=C_{1}\left(L^{m} \omega\right)(z)+C_{2}\left(L^{m} \bar{\omega}\right)(z)
$$

with the same constraint on the constants $C_{1}, C_{2}$ as before. 
REMARKS. For the derivative operator, Levin proves a more specific result and shows that

$$
g(z)=C_{1} \omega(z)+C_{2} \bar{\omega}(z) .
$$

While it is not necessary to obtain an explicit representation for $g$ itself in (2.9), one can easily do so when specializing the operator $L$. For example, when $L=$ $\varphi_{n}(D)=\prod_{i=1}^{n}\left(D+\lambda_{i} I\right)^{K_{\iota}}$, where $D=(d / d z), I=$ identity, the $K_{i}$ are positive integers and the numbers $\lambda_{1}, \ldots, \lambda_{n}$ are real and distinct, then equality at a point in Bernstein's inequality as in Lemma 3 implies

$$
g(z)=C_{1} \omega(z)+C_{2} \bar{\omega}(z)+\sum_{j=1}^{n} Q_{j}(z) \exp \left(-\lambda_{j} z\right),
$$

where $Q_{j}(z)$ is a polynomial of degree $\leqslant\left(K_{j}-1\right)$ for each $j=1, \ldots, n$. This is so since one can factor the operator

$$
\begin{aligned}
\left(D+\lambda_{1}\right)^{K_{1}} \cdots(D+ & \left.\lambda_{n}\right)^{K_{n}} g(z) \\
=\exp \left(-\lambda_{n} z\right) D^{K_{n}} & {\left[\exp \left(\left(\lambda_{n}-\lambda_{n-1}\right) z\right) D^{K_{n}}\right. \text { । }} \\
& \left.\times\left[\cdots \exp \left(\left(\lambda_{2}-\lambda_{1}\right) z\right) D^{K_{1}}\left[e^{\lambda_{1} z} g(z)\right] \cdots\right]\right]
\end{aligned}
$$

and use the known result of Levin. In this example, $\varphi_{n}(0) \neq 0$. Note also that

$$
\left(\varphi_{n}(D)\right)^{m} g(z)=C_{1}\left(\varphi_{n}(D)\right)^{m} \omega(z)+C_{2}\left(\varphi_{n}(D)\right)^{m} \bar{\omega}(z)
$$

for each $m=1,2,3, \ldots$.

3. Proof of the Theorem. By Lemma 1, for $\alpha$ real, we can express $f$ as

$$
f(z)=\cos (\lambda z+\alpha)-2 g(z)
$$

where either $g(z) \equiv 0$ or $g(z)$ is entire of exponential type $\tau \leqslant \lambda$ with only real zeros, is real on the real axis, satisfying $|g(z)| \leqslant 1$ on $(-\infty, \infty)$. Now, without loss of generality, assume $g(z) \neq 0$, for when $f(z) \equiv \cos (\lambda z+\alpha)$, the theorem has been proved in $[3,11]$. Consequently, let $(3.1)$ hold with $g$ having the properties stated above. Computations show that

$$
\begin{aligned}
& (\varphi(D))^{m} f(z)=2^{-1}\left[(\varphi(-i \lambda))^{m} \exp (-i \lambda z) \exp (-i \alpha)\right. \\
& \left.\quad+(\varphi(i \lambda))^{m} \exp (i \lambda z) \exp (i \alpha)\right]-2(\varphi(D))^{m} g(z)
\end{aligned}
$$

With $z=x+i y$, it is well known that $g$ satisfies

$$
|g(z)| \leqslant \exp (\tau|y|)=\left\{\begin{array}{lc}
|\exp (-i \tau z)|, & 0 \leqslant y<\infty, \\
|\exp (i \tau z)|, & -\infty<y<0
\end{array}\right.
$$

[1, Theorem 6.23]. In fact, by the Ahlfors-Heins theorem (cf. Boas [2, Theorem 1]) we have

$$
\lim _{r \rightarrow \infty} \log \left|g\left(r e^{i \theta}\right) / r=\tau\right| \sin (\theta) \mid
$$

for $\theta \neq 0, \pi$, where $\lambda \geqslant \tau=h_{g}(\pi / 2)=h_{g}(-\pi / 2)$ and $h_{g}$ denotes the indicator function of $g$. In particular, the indicator diagram for $g(z)$ is the interval $[-i \tau, i \tau]$. 
We now consider two cases: (a) $\tau<\lambda$; (b) $\tau=\lambda$. Since (3.3) holds, we have by Lemma 2 that

$$
\begin{aligned}
\left|(\varphi(D))^{m} g(z)\right| \leqslant & \begin{cases}\left|(\varphi(D))^{m}\left(e^{-i \tau z}\right)\right|, & \operatorname{Im}(z) \geqslant 0, \\
\left|(\varphi(D))^{m}\left(e^{i \tau z}\right)\right|, & \operatorname{Im}(z)<0,\end{cases} \\
& = \begin{cases}|(\varphi(-i \tau))|^{m}\left|e^{-i \tau z}\right|, & \operatorname{Im}(z) \geqslant 0, \\
|(\varphi(i \tau))|^{m}\left|e^{i \tau z}\right|, & \operatorname{Im}(z)<0 .\end{cases}
\end{aligned}
$$

Now, since $\varphi(\omega)$ is Laguerre-Pólya, computations show that $\mid \varphi($ it $) \mid$ is a strictly increasing function of $t$, so

$$
0 \neq|\varphi(i \tau)|^{m}<|\varphi(i \lambda)|^{m}
$$

for $0<\tau<\lambda$ and every $m=1,2,3, \ldots$ Letting $|\varphi(i \lambda)|=S$, we now proceed in the proof of the Theorem by arguing as in either [3] or [11]. For the sake of completeness, we give the details.

Let $\varphi(i \lambda) S \exp \left(i \mu_{1}\right), \varphi(-i \lambda)=S \exp \left(-i \mu_{2}\right)$. We then have

With $z$ restricted to a compact subset of the complex plane, we get, from (3.4),

$$
\left|2 S^{-m}(\varphi(D))^{m} g(z)\right|=o(1)
$$

as $m \rightarrow \infty$.

Now

$$
\begin{aligned}
& e^{i m \theta} S^{-m}(\varphi(D))^{m} f(z)=2^{-1} \exp \left(i m\left(\theta+\mu_{1}\right)\right) \exp (i \lambda z) \\
& \quad+2^{-1} \exp \left(i m\left(\theta-\mu_{2}\right)\right) \exp (-i \lambda z)-2 S^{-m} e^{i m \theta}(\varphi(D))^{m} g(z) .
\end{aligned}
$$

If $\theta=\left(\mu_{2}-\mu_{1}\right) / 2$ and $\beta=\left(\mu_{1}+\mu_{2}\right) / 2$, we obtain

$$
\begin{aligned}
e^{i m \theta} S^{-m}(\varphi(D))^{m} f(z)= & 2^{-1} \exp (-i m \beta) \exp (-i \lambda z) \\
& +2^{-1} \exp (i m \beta) \exp (i \lambda z)+o(1) \\
= & Q_{m}(z)+o(1)
\end{aligned}
$$

with $Q_{m}(z)$ as indicated. If $\beta=(p / q) \pi$ is a rational multiple of $\pi$, then $Q_{m}(z)$ is periodic in $m$ with period $q$ (up to factors independent of $z$ ). Therefore the final set consists of the zeros of $Q_{m}(z), Q_{m+1}(z), \ldots, Q_{m+q-1}(z)$ and is a discrete subset of the line $\operatorname{Im}(z)=0$, i.e. the real axis. On the other hand, when $\beta$ is an irrational multiple of $\pi$, the final set consists of limit points of the zeros of $Q_{m}(z)$, that is, limits of points of the form

$$
z=(2 \lambda)^{-1}\{2 m \beta+(2 K+1) \pi\}
$$

with $K=0, \pm 1, \pm 2, \ldots$. The points in (3.9) are dense on the real axis, making the final set the real axis.

For case (b) where $\tau=\lambda$, we argue as follows. When strict inequality holds in (3.4) for all $m=1,2,3, \ldots$ then the preceding argument applies to establish the result. So suppose $\tau=\lambda$ and there exists a positive integer $M$ for which equality holds in (3.4). We first present the argument for the derivative operator because it is 
so simple and follow it with the general case. When equality holds in (3.4) for some $M$, by Lemma 3 and subsequent remarks, we have

$$
g(z)=C_{1} \exp (-i \lambda z)+C_{2} \exp (i \lambda z),
$$

for complex numbers $C_{1}, C_{2}$ satisfying $\left|C_{1}\right|+\left|C_{2}\right|=1$. Since $g$ is real on the real axis, we have $C_{2}=\bar{C}_{1}$. So $\left|C_{1}\right|=\left|C_{2}\right|=2^{-1}$. Let us say that $C_{1}=2^{-1} \exp (-i \gamma)$, $C_{2}=2^{-1} \exp (i \gamma)$ for some real $\gamma$. Therefore

$$
\begin{aligned}
f(z)= & \left(2^{-1} \exp (-i \alpha)-\exp (-i \gamma)\right) \exp (-i \lambda z) \\
& +\left(2^{-1} \exp (i \alpha)-\exp (i \gamma)\right) \exp (i \lambda z) .
\end{aligned}
$$

The coefficients of $\exp ( \pm i \lambda z)$ are both nonzero. Thus the final set of $f$ is a discrete subset of the real axis, as was established by the author in [10].

In the general case, when equality holds in (3.4) for some positive integer $M$, Lemma 3 gives for all integers $m \geqslant M$,

$$
\begin{aligned}
\left((\varphi(D))^{m} g\right)(z) & =C_{1}(\varphi(D))^{m} \exp (-i \lambda z)+C_{2}(\varphi(D))^{m} \exp (i \lambda z) \\
& =C_{1}(\varphi(-i \lambda))^{m} \exp (-i \lambda z)+C_{2}(\varphi(i \lambda))^{m} \exp (i \lambda z)
\end{aligned}
$$

where $C_{1}$ and $C_{2}$ are complex constants satisfying $\left|C_{1}\right|+\left|C_{2}\right|=1$. Now, since $\varphi(w)=\Sigma_{k} b_{k} w^{k}$ is Laguerre-Pólya and so is real valued on the real axis, the coefficients $b_{k}$ are real. Since $g(z)$ is real on the real axis, so are $g^{(k)}(z)$ for $k=1,2,3, \ldots$ and, consequently, so are

$$
\varphi(D) g(z)=\sum_{k} b_{k} g^{(k)}(z)
$$

and $(\varphi(D))^{m} g(z)$ real on the real axis for $m=2,3, \ldots$ Therefore, in (3.10) we have

$$
C_{1}(\varphi(-i \lambda))^{m}=\overline{C_{2}(\varphi(i \lambda))^{m}}
$$

for each $m$ so $C_{1}=\bar{C}_{2}$. Since $\left|C_{1}\right|+\left|C_{2}\right|=1$ we have $C_{1}=2^{-1} \exp (-i \gamma), C_{2}=$ $2^{-1} \exp (i \gamma)$ for some real $\gamma$. As a result from (3.10) we have

$$
\begin{aligned}
(\varphi(D))^{m} f(z) & =(\varphi(D))^{m}\left\{2^{-1} \exp (-i \alpha) \exp (-i \lambda z)+2^{-1} \exp (i \alpha) \exp (i \lambda z)\right\} \\
& -2\left((\varphi(D))^{m} g\right)(z) \\
= & \left(2^{-1} \exp (-i \alpha)-\exp (-i \gamma)\right)(\varphi(-i \lambda))^{m} \exp (-i \lambda z) \\
& +\left(2^{-1} \exp (i \alpha)-\exp (i \gamma)\right)(\varphi(i \lambda))^{m} \exp (i \lambda z)-2\left((\varphi(D))^{m} g\right)(z) \\
= & \bar{d}_{1}(\varphi(-i \lambda))^{m} \exp (-i \lambda z)+d_{1}(\varphi(i \lambda))^{m} \exp (i \lambda z)-2\left((\varphi(D))^{m} g\right)(z)
\end{aligned}
$$

with $d_{1}$ as indicated. The coefficients of $\exp ( \pm i \lambda z)$ are both nonzero. Hence, by appealing to Theorem 1 of [11] or by suitably modifying the argument given in case (a), we have proved the Theorem.

ACKNOWLEDGEMENT. The present work was done while the author was visiting the University of Wisconsin, Madison. The author thanks S. Hellerstein and D. Shea for their hospitality. 


\section{REFERENCES}

1. R. P. Boas, Entire functions, Academic Press, New York, 1954.

2. Asvmptotic properties of functions of exponential type, Duke Math J. 20 (1953), 433-448.

3. R. P. Boas and C. L. Prather, Final sets for operators on finite Fourier transforms, Houston Math. J. 5 (1979), 29-36.

4. R. Duffin and A. C. Schaeffer, Some properties of functions of exponential type, Bull. Amer. Math. Soc. 44 (1938), 236-240.

5. A. Edrei, On a conjecture of Pólya concerning the zeros of successive derivatives, Scripta Math. 22 (1956), 31-44; ibid. 23 (1957), 106-121.

6. R. Gethner, A Pólya "schire" theorem for entire functions, Ph. D. Thesis, Univ. of Wisconsin, Madison, 1982.

7. B. Ja. Levin, Distribution of zeros of entire functions, 2nd ed., Amer. Math. Soc. Transl. (2) 5 (1980).

8. G. Pólya, On the zeros of the derivatives of a function and its analytic character, Bull. Amer. Math. Soc. 49 (1943), 178-191.

9. Ü Über die Realität der Nullstellen fast aller Ableitungen gewisser ganzer Funktionen, Math. Ann. 144 (1937), 622-634.

10. C. Prather, On the zeros of derivatives of balanced trigonometric polynomials, Pacific J. Math. 81 (1979), 516-523.

11. On some new and old theorems, on final sets, Houston Math. J. 7 (1981), 407-430.

12. Final sets for operators on classes of entire functions representable as a Fourier integral, J. Math. Anal. Appl. 82 (1981), 200-220.

13. Zeros of operators on functions and their analytic character, Rocky Mountain J. Math. (to appear).

Department of Mathematics, Virginia Polytechnic Institute and State University, BlacksBURG, VIRGINIA 24061-4097 